\title{
FIRST LOOK AT THE TIME-DEPENDENT $C P$ VIOLATION USING EARLY BELLE II DATA
}

\author{
Vladimir Chekelian ${ }^{a}$ \\ Max Planck Institute for Physics, Munich, Germany
}

Abstract. The Belle II experiment at the SuperKEKB energy-asymmetric $e^{+} e^{-}$ collider started the first physics run in March 2019 at a center of mass energy corresponding to the mass of the $\Upsilon(4 S)$. A first look at the time-dependent $C P$ violation is presented, utilising an early data set comprising $2.6 \mathrm{fb}^{-1}$ of total luminosity. A new concept of time-dependent fits based on a weighting technique applied to an assistive MC sample of events is presented as well.

\section{Introduction}

The mixing-induced $C P$ violation $(C P V)$ is one of the physics subjects, which could shed light on physics beyond the Standard Model, by making precise measurements of the unitarity triangle. This is an important part of the motivation for the new high luminosity experiment Belle II [1] at the SuperKEKB energy-asymmetric $e^{+} e^{-}$collider. The design luminosity of the new machine is $8 \times 10^{35} \mathrm{~cm}^{-2} \mathrm{~s}^{-1}$, and the Belle II experiment aims to record $50 \mathrm{ab}^{-1}$ of data, a factor of 50 more than recorded by its predecessor, the Belle experiment at the $B$-factory KEKB.

The first steps of the time-dependent $C P V$ analysis using an early data set of $2.6 \mathrm{fb}^{-1}$ total luminosity, collected in the spring of 2019 , are reported here. One neutral $B$-meson from the decay $\Upsilon(4 S) \rightarrow B^{0} \bar{B}^{0}$ is reconstructed in the $J / \psi K_{S}^{0}$ $C P$-eigenstate decay channel. Then, the $C P V$ parameters and the CKM angle $\phi_{1}(\beta)$ are determined in the fit of the time difference, $\Delta t=t_{B_{C P}^{0}}-t_{B_{\mathrm{tag}}^{0}}$, between the decay times of the reconstructed signal, $B_{C P}^{0}$, and the accompanying $B$ meson, $B_{\text {tag }}^{0}$. The flavor of the latter is identified (tagged) from the decay products to be either $B^{0}(q=1)$ or $\bar{B}^{0}(q=-1)$. At this early stage we report on the observation of $B$-decays in the "golden channel" $B^{0} \rightarrow J / \psi K_{S}^{0}$ and on the measurement of $B^{0}-\bar{B}^{0}$ mixing in semi-leptonic $B^{0}$-decays.

In this paper a new concept of time-dependent fits based on a weighting technique applied to an assistive sample of simulated MC events is described for the first time, see also [2].

\section{Observation of $B^{0} \rightarrow J / \psi K_{S}^{0}$ decay and of $B^{0}-\bar{B}^{0}$ mixing}

The "golden decay channel" $B^{0} \rightarrow J / \psi K_{S}^{0}$ is reconstructed using $J / \psi$-decays to $\mu^{+} \mu^{-}$or $e^{+} e^{-}$pairs and $K_{S}^{0}$-candidates, reconstructed from two tracks with opposite charge and common origin in space displaced from the $B$-decay vertex.

\footnotetext{
${ }^{a}$ E-mail: shekeln@mpp.mpg.de
} 
The numbers of signal and background events in data are determined in a twodimensional unbinned maximum likelihood fit using the beam constrained mass $M_{\mathrm{bc}}=\sqrt{E_{\text {beam }}^{2}-p_{\mathrm{cms}}^{2}}$ and the energy difference $\Delta E=E_{\text {beam }}-E_{\mathrm{cms}}$. Here, $E_{\text {beam }}$ is the cms beam energy, and $p_{\mathrm{cms}}$ and $E_{\mathrm{cms}}$ are the measured momentum and energy of the $B$-candidate in the rest frame of the $\Upsilon(4 S)$. The $M_{\mathrm{bc}}$ and $\Delta E$ distributions of selected $B^{0} \rightarrow J / \psi K_{S}^{0}$ events are shown in Fig. 1 together with fit results. The visible $B^{0} \rightarrow J / \psi K_{S}^{0}$ yield is found to be $26.9 \pm 5.2$ events.
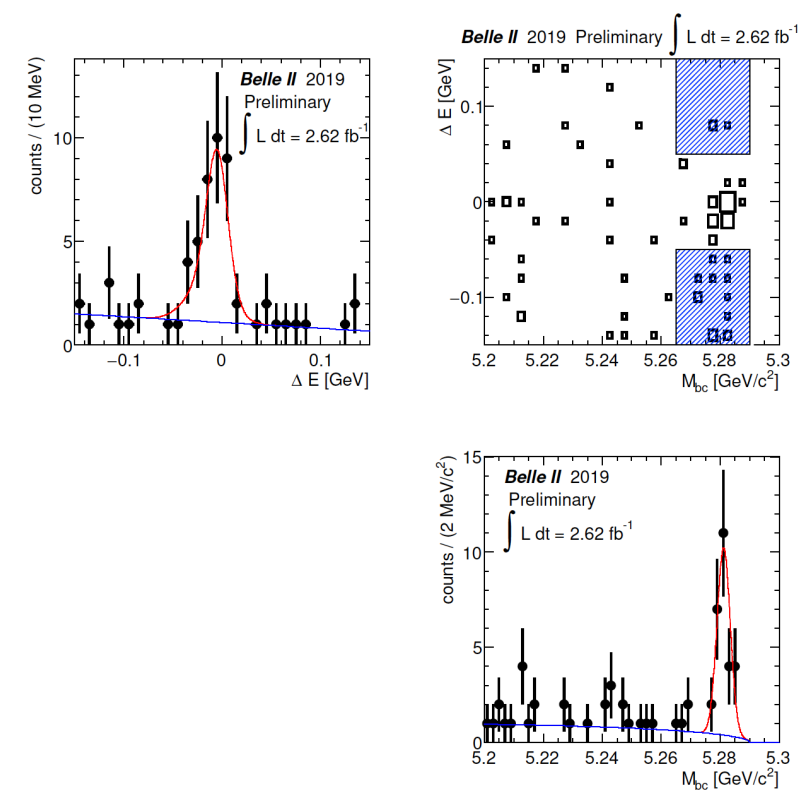

Figure 1: $M_{\mathrm{bc}}$ and $\Delta E$ distributions for $B^{0} \rightarrow J / \psi K_{S}^{0}$ events. Fit results are also shown. Shaded regions are excluded from the fit to remove mainly the $B^{0} \rightarrow J / \psi K^{* 0}$ background.

This event sample is too small to perform a time-dependent $C P V$ analysis. The potential of the apparatus in this respect is however demonstrated by the measurement of $B^{0}-\bar{B}^{0}$ mixing. Due to the asymmetric energies of the $e^{+}$and $e^{-}$beams, the $\Upsilon(4 S)$ is produced with a Lorentz boost of $\beta \gamma=0.28$. The $B \bar{B}$ pairs are produced almost at rest in the $\Upsilon(4 S)$ frame, and the decay time difference can be determined from the space separation of the $B^{0}$-decay vertices $\Delta t \approx \Delta z /(\beta \gamma c)$. The high precision of the vertex position measurements of $14.2 \pm 0.1 \mu \mathrm{m}$ is proven in data using two-track Bhabha events. This precision is achieved by using the pixel vertex detector (PXD) composed of pixels of $50 \times 55 \times 75 \mu \mathrm{m}^{3}$ size, located at $14 \mathrm{~mm}$ from the beam axis.

The mixing rate is measured using events with flavor information available for both $B$-mesons decaying semi-leptonically. Events with opposite charges 
of the leptons correspond to $B^{0} \bar{B}^{0}$ states without mixing. This "unmixed" sample comprises $1642 \pm 113$ events. The same charges of the leptons indicate that one of the $B^{0}$-mesons changed flavor due to $B^{0}-\bar{B}^{0}$ mixing. $253 \pm 45$ "mixed" events are found. The fraction of mixed events corrected for efficiency, $(17.3 \pm 3.6) \%$, is in agreement with the expectation of $18.6 \%$. The evolution of the fraction of unmixed events as a function of $|\Delta t|$ in Fig. 2 (left) is consistent with the expected oscillation pattern of the $B^{0}-\bar{B}^{0}$ mixing, assuming world averages for the $B^{0}$-lifetime $(\tau)$ and for the $B^{0}-\bar{B}^{0}$ mixing parameter $\left(\Delta m_{d}\right)$.
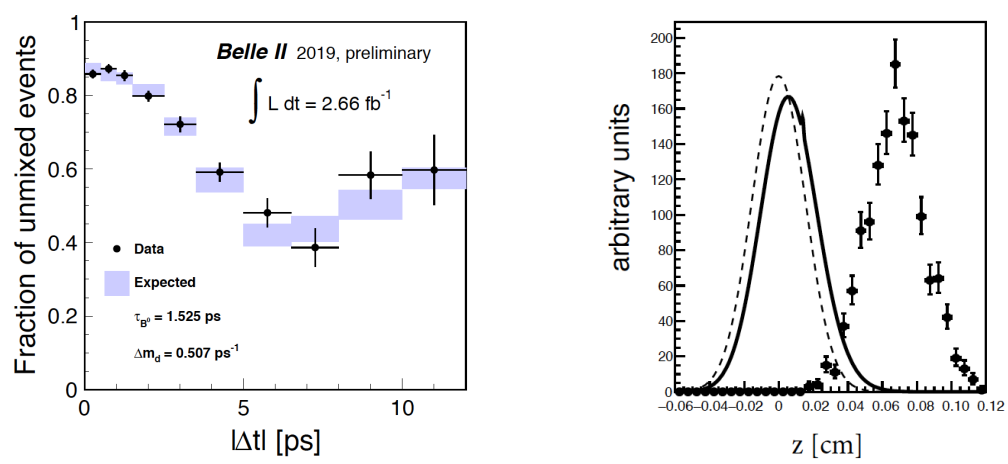

Figure 2: Left: Fraction of unmixed events as a function of $|\Delta t|$ for $B^{0} \rightarrow D^{* 0} l \nu$ events. Right: Simulation of the decay $z$-positions of the $Y(4 S)$ (dashed line), of $B_{\mathrm{tag}}^{0}$ if it decays first, i.e. $\Delta t>0$, (solid line) and of $B_{\mathrm{tag}}^{0}$ for $\Delta t$ from -8 to $-6.7 \mathrm{ps}$ (points).

\section{A new concept of time-dependent fits at Belle II}

In view of new challenges related to the target statistics and the high precision of Belle II, an alternative paradigm for time-dependent analyses is desirable. For example, the size of the beam spot is much smaller than the decay length of $B^{0}$-mesons on the tag side (700 $\mu \mathrm{m}$ and more) for very negative $\Delta t$-values, see Fig. 2 (right). This could cause a variation of the quality of the $B_{\text {tag }}$ vertex reconstruction and a dependence of the $\Delta t$ resolution function on $\Delta t_{\text {true }}$ and on the underlying physics parameters. In the traditional time-dependent analyses at Belle and BaBar [3], the convolution of theory input and the $\Delta t$ resolution function is done analytically assuming their factorisation. To overcome these complications, the new concept [2] is utilising a weighting technique applied to an assistive MC simulated using GEANT and the best knowledge of the detector properties. By that the more precise convolution is taken from the simulation without the need of an intermediate analytical approximation of the $\Delta t$ resolution function. The basic principles of the concept are the following:

- an unbinned maximum likelihood fit is used to determine all physics and smearing parameters, with the likelihood $L=\prod_{i} P_{e v}^{i}(\Delta t)$; 

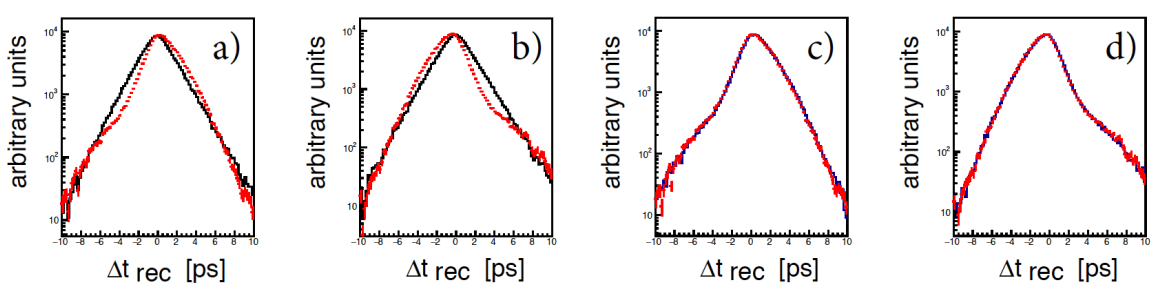

Figure 3: The reconstructed $\Delta t_{\mathrm{rec}}$ distributions for $q=1(\mathrm{a}, \mathrm{c})$ and $q=-1$ (b, d) for $B^{0} \rightarrow J / \Psi K_{S}^{0}$ MC simulated without (black in a,b) and with (blue in c, d) $C P$ violation.

The MC sample without $C P V$, re-weighted to account for $C P$ violation, is shown in red.

- the event probabilities $P_{e v}^{i}(\Delta t)$ are calculated numerically using a dedicated assistive MC sample simulated for the chosen signal $B$-decays;

- the physics parameters $\left(\tau, \Delta m_{d}\right.$ and parameters $A$ and $S$ for the direct and mixing-induced $C P V$ ) are varied in the fit by weighting of the events in the assistive $\mathrm{MC}$ sample in such a way that the re-weighted sample is equivalent to a new simulation with new values of physics parameters;

- similarly, re-weighting of the assistive MC sample is applied for additional smearing of $\Delta t$ to downgrade the detector response, with the goal to reproduce in simulation the detector properties of the data;

The additional $\Delta t$ smearing of the assistive $\mathrm{MC}$ is expected to be pretty much the same for all channels in the fit, since the $\Delta t$ resolution is dominated by the quality of the $B_{\text {tag }}$ vertex reconstruction, which is common for all channels. Details of the weighting procedures are discussed in the next two sections.

\subsection{Re-weighting of an assistive MC sample instead of new physics simulation}

The theory input for generation and simulation of events is given by the following probability function depending on the decay time of the $B^{0}$, which decays first, $t_{B_{\text {first }}^{0}}$, and on the time difference of $B_{C P}^{0}$ and $B_{\text {tag }}^{0}$ decays:

$$
P_{t h}=\frac{e^{\left(-t_{B_{f i r s t}^{0}} / \tau\right)}}{4 \tau} \frac{e^{(-|\Delta t| / \tau)}}{4 \tau}\left\{1+q\left[A \cdot \cos \left(\Delta m_{d} \Delta t\right)+S \cdot \sin \left(\Delta m_{d} \Delta t\right)\right]\right\}
$$

Re-weighting of the events in the simulated MC sample by the ratio of probabilities calculated using a new set of physics parameters and of the set used during simulation, results in a new MC sample, which is fully equivalent to a new simulation with the new set. This is demonstrated in Fig. 3, where red histograms obtained after re-weighting coincide in c) and d) with the blue histograms corresponding to the simulation with the new set of parameters. 


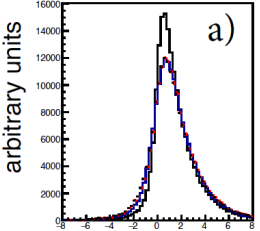

$\Delta \mathrm{t}$ rec [ps]

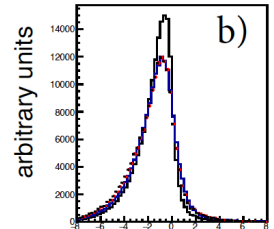

$\Delta \mathrm{t}$ rec $[\mathrm{ps}]$

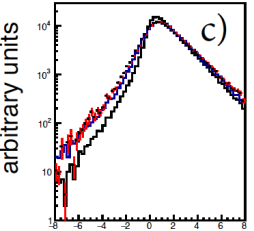

$\Delta \mathrm{t}$ rec $[\mathrm{ps}]$

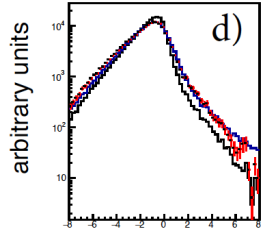

$\Delta \mathrm{t}$ rec $[\mathrm{ps}]$

Figure 4: The $\Delta t_{\mathrm{rec}}$ distributions for positive (a, c) and negative (b, d) $\Delta t_{\text {true }}$ in the $B^{0} \rightarrow J / \Psi K_{S}^{0} \mathrm{MC}$ sample without smearing (black), with smearing (blue) and smeared by weighting (red). The vertical scales are linear in $(\mathrm{a}, \mathrm{b})$ and logarithmic in $(\mathrm{c}, \mathrm{d})$.

\subsection{Smearing of an assistive $M C$ sample by weighting of the $M C$ events}

The most straightforward and very efficient way to account for differences between data and $\mathrm{MC}$ in the detector response is an additional Gaussian smearing of the reconstructed $\Delta t_{\mathrm{rec}}$ in $\mathrm{MC}, \Delta t_{\mathrm{rec}}^{\text {smear }}=\Delta t_{\mathrm{rec}}+G\left(0, \alpha_{\text {smear }} \cdot \delta(\Delta t)\right)$, scaled with the uncertainty of $\Delta t_{\mathrm{rec}}, \delta\left(\Delta t_{\mathrm{rec}}\right)$. This Gaussian smearing can be applied before the fit, however, it is not suitable for iterations in the fitting procedure. Since the resolution function, $P\left(\Delta t_{\mathrm{rec}}-\Delta t_{\text {true }}\right)$, is often approximated by a sum of three Gaussian functions, one can do smearing by weighting of the MC events with the ratio of probabilities of the deviation $\Delta t_{\mathrm{rec}}-\Delta t_{\text {true }}$ for modified and initial resolution functions in $\mathrm{MC}$, where the modification is given by replacing all Gauss sigma-parameters, $\sigma_{i}$, by $\sigma_{i}^{\text {smear }}=\sqrt{\sigma_{i}^{2}+\left(\alpha_{\text {smear }} \cdot \delta(\Delta t)\right)^{2}}$. In Fig. 4 it is demonstrated for the $B^{0} \rightarrow J / \Psi K_{S}^{0}$ signal $\mathrm{MC}$ and $\alpha_{\text {smear }}=2$, that both ways (blue and red histograms) are almost identical. Consequently, the factor $\alpha_{\text {smear }}$ in the smearing by weighting can be used as an additional free parameter in the fitting procedure.

\section{Conclusions}

The first $2.6 \mathrm{fb}^{-1}$ of Belle II data collected in 2019 are used to observe the decays $B^{0} \rightarrow J / \psi K_{S}^{0}$ - the "golden channel" for measurements of time-dependent $C P$ violation. The feasibility of time-dependent analyses is demonstrated by the measurement of $B^{0}-\bar{B}^{0}$ mixing using events where both $B^{0}$-mesons decay semi-leptonically. In view of the new challenges related to the target statistics and the high precision of Belle II, an alternative paradigm for time-dependent analyses based on the weighting of an assistive MC sample is developed.

[1] T. Abe et al., Belle II Technical Design Report, [arXiv:1011.0352] (2010).

[2] Vladimir Chekelian, The MPI Concept of Time-Dependent Fits at Belle II, The xFitter Workshop, Minsk, 18-20 March 2019, https:

//indico.desy.de/indico/event/22011/session/7/contribution/24/material/slides/0.pdf

[3] A. J. Bevan et al., Eur. Phys. J. C74 (2014) 3026, [arXiv:1406.6311]. 